\title{
Thyroid hormone responsive (THRSP) promotes the synthesis of medium-chain fatty acids in goat mammary epithelial cells
}

\author{
D. W. Yao, ${ }^{*}$ J. Luo, ${ }^{1}$ Q. Y. He, ${ }^{*}$ M. Wu, ${ }^{*}$ H. B. Shi, $†$ H. Wang, ${ }^{*}$ M. Wang, ${ }^{*}$ H. F. Xu, ${ }^{*}$ and J. J. Loor ${ }^{1}$ \\ *Shaanxi Key Laboratory of Molecular Biology for Agriculture, College of Animal Science and Technology, Northwest A\&F University, Yangling, \\ Shaanxi, P. R. China 712100 \\ †College of Animal Sciences, Zhejiang Sci-Tech University, Hangzhou, P. R. China 310058 \\ ¥Mammalian NutriPhysioGenomics, Department of Animal Sciences and Division of Nutritional Sciences, University of Illinois, Urbana 61801
}

\begin{abstract}
In nonruminants, thyroid hormone responsive (THRSP) is a crucial protein for cellular de novo lipogenesis. However, the role of THRSP in regulating the synthesis of milk fatty acid composition in goat mammary gland remains unknown. In the present study, we compared gene expression of THRSP among different goat tissues. Results revealed that THRSP had the highest expression in subcutaneous fat, and expression was higher during lactation compared with the dry period. Overexpression of THRSP upregulated the expression of fatty acid synthase $(F A S N)$, stearoyl-coenzyme A desaturase 1 (SCD1), diacylglycerol acyltransferase 2 (DGAT2), and glycerol-3-phosphate acyltransferase $(G P A M)$ in goat mammary epithelial cells. In contrast, overexpression of THRSP led to downregulation of thrombospondin receptor $(C D 36)$ and had no effect on the expression of acetyl-coenzyme A carboxylase $\alpha$ $(A C A C A)$ and sterol regulatory element binding transcription factor1 (SREBF1). In addition, overexpressing THRSP in vitro resulted in a significant increase in triacylglycerol (TAG) concentration and the concentrations of C12:0 and C14:0. Taken together, these results highlight an important role of THRSP in regulating lipogenesis in goat mammary epithelial cells.
\end{abstract}

Key words: fatty acid composition, goat, milk fat, dairy nutrition

\section{INTRODUCTION}

Goat milk is a significant food and nutrient source for people worldwide. Fat composition is one of the most valuable components in terms of goat milk quality. Compared with cow milk, goat milk contains a much higher proportion of short- and medium-chain fatty

\footnotetext{
Received November 12, 2015.

Accepted December 14, 2015

${ }^{1}$ Corresponding authors: luojun@nwsuaf.edu.cn and jloor@illinois.
} edu acids (MCFA; Haenlein and Caccese, 1984; Haenlein, 2004; Kompan and Komprej, 2012), which endows goat milk a special function in the treatment of some human metabolic disorders (Babayan, 1981; Williams, 2000). The higher content of beneficial unsaturated fatty acids (FA; e.g., cis-9-C18:1 and cis-9, trans-11-C18:2) in goat than in cow milk further enhances its nutritive value (Hinders, 1999; Alférez et al., 2001; Kompan and Komprej, 2012). Of the total FA in milk fat, 40 to $50 \%$ of short- and medium-chain FA (C4:0 to C16:0) arise from de novo synthesis in the mammary gland (Mather and Keenan, 1998). The FA are esterified into triacylglycerol (TAG) within mammary epithelial cells to form a complex mixture of lipid droplets that can be secreted during the milking process (Clegg et al., 2001).

Thyroid hormone responsive (THRSP) was first identified as a key protein in studies evaluating the effect of thyroid hormone on rat hepatic mRNA expression (Seelig et al., 1981). More recent work in rodents demonstrated that in liver or adipose tissue it is strongly activated by lipogenic stimuli such as feeding of high-carbohydrate diets (LaFave et al., 2006). It is now known that THRSP and its homolog Spot-related protein (MIG12, Spot14-R) can form heterodimers to modulate FA synthesis (Colbert et al., 2010).

In rodents, a microarray study of gene expression in the mouse mammary gland revealed that Thrsp was markedly upregulated upon the onset of lactation $(\mathrm{Ru}-$ dolph et al., 2003). Furthermore, Thrsp-null mice had a marked reduction of MCFA and TAG concentrations in milk (Zhu et al., 2005). More direct evidence for the involvement of THRSP in FA synthesis was obtained using a THRSP-knockdown model that led to reduced mammary cancer cell incorporation of labeled acetate into lipids (Martel et al., 2006). Although THRSP seems to play a role in lipogenesis, the fact that a global Thrsp deletion increased hepatic lipogenesis in vivo in response to acute thyroid hormone and a lipogenic diet suggests that, at least in mouse, THRSP might not be required for induction of lipogenesis (Zhu et al., 2001). The lipogenic response via THRSP might be related 
to its interactions with Spot14-R; for example, its association with Spot14-R and the upstream effect on activation of acetyl-CoA carboxylase $\alpha$ (ACACA). In vivo studies demonstrated that ACACA is regulated by Spot14-R via promoting its polymerization and greater enzymatic activity (Zhu et al., 2005; Kim et al., 2010). Consequently, these findings led us to conclude that the effect of THRSP on de novo FA synthesis depends on the presence of Spot14-R in different tissues or cell types.

Compared with rodents, the question of whether THRSP is essential in de novo FA synthesis and FA composition in ruminant mammary cells has not been fully explored. The main goal of this study was to examine the extent to which overexpression of THRSP in goat mammary epithelial cells (GMEC) could induce expression of key lipogenic target genes as well as TAG synthesis. In addition, we used FA analysis to determine the extent to which THRSP can alter the content of medium-chain and unsaturated FA known to originate from de novo synthesis.

\section{MATERIALS AND METHODS}

The Animal Care and Use Committee of the Northwest A\&F University (China) approved all procedures and experiments.

\section{Animals and Tissue Samples}

Xinong Saanen dairy goats used in this research were selected from the experimental farm at the Northwest A\&F University, Shaanxi, China. Three healthy, nonlactating Xinong Saanen dairy goats (5 yr old, fourth parity) raised in confinement were slaughtered for tissue sampling, including subcutaneous fat, muscle, liver, lung, mammary gland, rumen, small intestine, kidney, heart, and spleen. In addition, mammary gland tissue samples were collected from 3 healthy Xinong Saanen dairy goats (6 yr old, fifth parity) at peak lactation. The average milk yield of the 3 goats was $3.0 \mathrm{~kg} / \mathrm{d}$ during peak lactation. The average percentages of fat, protein, lactose, and total solids of the milk was 3.94, $2.99,4.63$, and 12.28 , respectively. All tissue samples were obtained under sterile conditions within $20 \mathrm{~min}$ and rinsed with DEPC-treated (Sigma, St. Louis, MO) sterile water and then frozen in liquid nitrogen instantly until RNA extraction.

\section{Adenovirus Generation}

The procedure for adenovirus generation was previously described (Shi et al., 2013). Briefly, the cDNA sequence of dairy goat THRSP (Gene ID: KU051565) was subcloned into the pAdTrack-CMV shuttle vector between the BglII and SalI (New England BioLabs Inc., Ipswich, MA) restriction sites to generate the pAdTrack-CMV-THRSP vector. Then, this vector was linearized by PmeI (New England BioLabs Inc.) and inserted into an adenoviral vector (pAdEasy-1) to generate adenoviral plasmids in BJ5183 cells. The linearized adenoviral plasmid by PacI (New England BioLabs Inc.) was transfected into 293A cells to generate the adenovirus with THRSP (Ad-THRSP) using a commercial system (AdEasy, Stratagene, La Jolla, $\mathrm{CA}$ ). The adenovirus containing green fluorescent protein (Ad-GFP) was used as a negative control and was a gift from Zhijie Chang (Tsinghua University, Beijing, China).

\section{Total RNA Extraction and Quantitative Real-Time PCR}

Total RNA was extracted from tissues using Trizol reagent (Invitrogen Corp., Carlsbad, CA). The cDNA was synthesized from $0.5 \mu \mathrm{g}$ of purified total RNA using PrimeScript RT kit with gDNA Eraser (Perfect Real time, Takara Bio Inc., Otsu, Japan) to remove genomic DNA contamination. The relative expression of genes in tissues and GMEC were detected using SYBR Green (SYBR Premix Ex TaqII, Perfect Real Time, Takara Bio Inc.) on a CFX96 Real-Time PCR Detection System (Bio-Rad, Hercules, CA) according to the manufacturer's instructions.

The genes evaluated were fatty acid synthase $(\boldsymbol{F A S N}), A C A C A$, sterol regulatory element binding transcription factor 1 ( $\boldsymbol{S R E B F 1 ) , ~ s t e a r o y l - C o A ~}$ desaturase 1 ( $\boldsymbol{S C D 1}$ ), diacylglycerol acyltransferase 2 (DGA T2), glycerol-3-phosphate acyltransferase $(\boldsymbol{G P A M})$, thrombospondin receptor $(\boldsymbol{C D} \mathbf{3 6})$, perilipin 2 (PLIN2), fatty acid binding protein 3 (FABP3). The data were normalized to ubiquitously expressed transcript $(\boldsymbol{U} \boldsymbol{X} \boldsymbol{T})$, mitochondrial ribosomal protein L39 (MRPL39), and ribosomal protein S9 (RPS9) (Ramunno et al., 2005; Bionaz and Loor, 2007). Every sample was analyzed in triplicate. Primer sequences for quantitative real-time PCR are listed in Table 1.

\section{Cell Culture and Treatment}

The GMEC were obtained from peak-lactation Xinong Saanen dairy goats and cultured as previously described (Wang et al., 2010; Shi et al., 2014; Li et al., 2015). To promote lactogenesis, GMEC were cultured in a basal medium supplemented with prolactin $(2 \mu \mathrm{g} /$ $\mathrm{mL}$, Sigma-Aldrich, St. Louis, MO) for $48 \mathrm{~h}$ before 
initial experiments, as described previously (Peterson et al., 2004; Kadegowda et al., 2009). The GMEC were seeded in 12-well cell culture clusters (Nunc, Thermo Fisher Scientific, Waltham, MA) for $24 \mathrm{~h}$ and infected with Ad-GFP or Ad-THRSP at approximately 80 to $90 \%$ confluence. Then, GMEC were harvested at 48 $\mathrm{h}$ in fetal bovine serum-free medium with sodium acetate $(5 \mathrm{mmol} / \mathrm{L}$, Sigma-Aldrich) and BSA (1 g/L, Sigma-Aldrich) for RNA extraction, TAG assay, and FA extraction.

\section{FA Extraction and Analysis}

Total FA were extracted from approximately 100 mg of cells. After incubation with virus for $48 \mathrm{~h}$, cells were washed 3 times with PBS and then scraped off the culture dish using a $2-\mathrm{mL}$ aliquot of $2.5 \%$ ( $\mathrm{vol} / \mathrm{vol}$ ) vitriol:methanol. Then, GMEC were transferred into a clean tube according to protocols described previously (Folch et al., 1957). Methylated lipid samples were analyzed using a GC-MS equipment (Agilent Technologies, Santa Clara, CA) installed with an HP-5 column (60 m $\times 0.25 \mathrm{~mm}$ i.d. $\times 0.25 \mu \mathrm{m}$ film thickness) following a published procedure from our laboratory (Wang et al., 2012). The relative proportions of FA were calculated as percentages of the total peak areas that could be detected.

\section{Cellular TAG Assays}

Intracellular TAG concentration assay was conducted as previously described (Kang et al., 2015). Briefly, after infection with adenovirus for $48 \mathrm{~h}$, GMEC were rinsed twice with PBS and then harvested in lysis buffer $(50$ $\mathrm{m} M$ Tris- $\mathrm{HCl}, \mathrm{pH} 7.4,150 \mathrm{~m} M \mathrm{NaCl}, 1 \%$ Triton X-100). A TAG kit (GPO-POD; Applygen Technologies Inc., Beijing, China) was used following the manufacturer's instructions to measure the intracellular TAG content. Total protein concentrations were measured using the BCA protein assay kit (Thermo Fisher Scientific) and measured on a NanoDrop ND 2000 (Thermo Fisher Scientific). The TAG concentrations were calculated by normalizing to the total cellular protein abundance and are reported as micrograms per milligram of protein.

\section{Statistical Analysis}

The results were analyzed using SPSS 19.0 (SPSS Inc., Chicago, IL) and reported as means \pm standard error of the means (SEM) of at least 3 independent experiments. The quantitative real-time PCR data were analyzed using the $2^{-\Delta \Delta \mathrm{Ct}}$ method relative to the control, where Ct is the cycle threshold. Relative content of each FA is reported as a portion of the total FA, and the desaturation index was calculated as the

Table 1. Characteristics of primers used and efficiency of the quantitative real-time PCR reaction $(\mathrm{F}=$ forward; $\mathrm{R}=$ reverse $)$

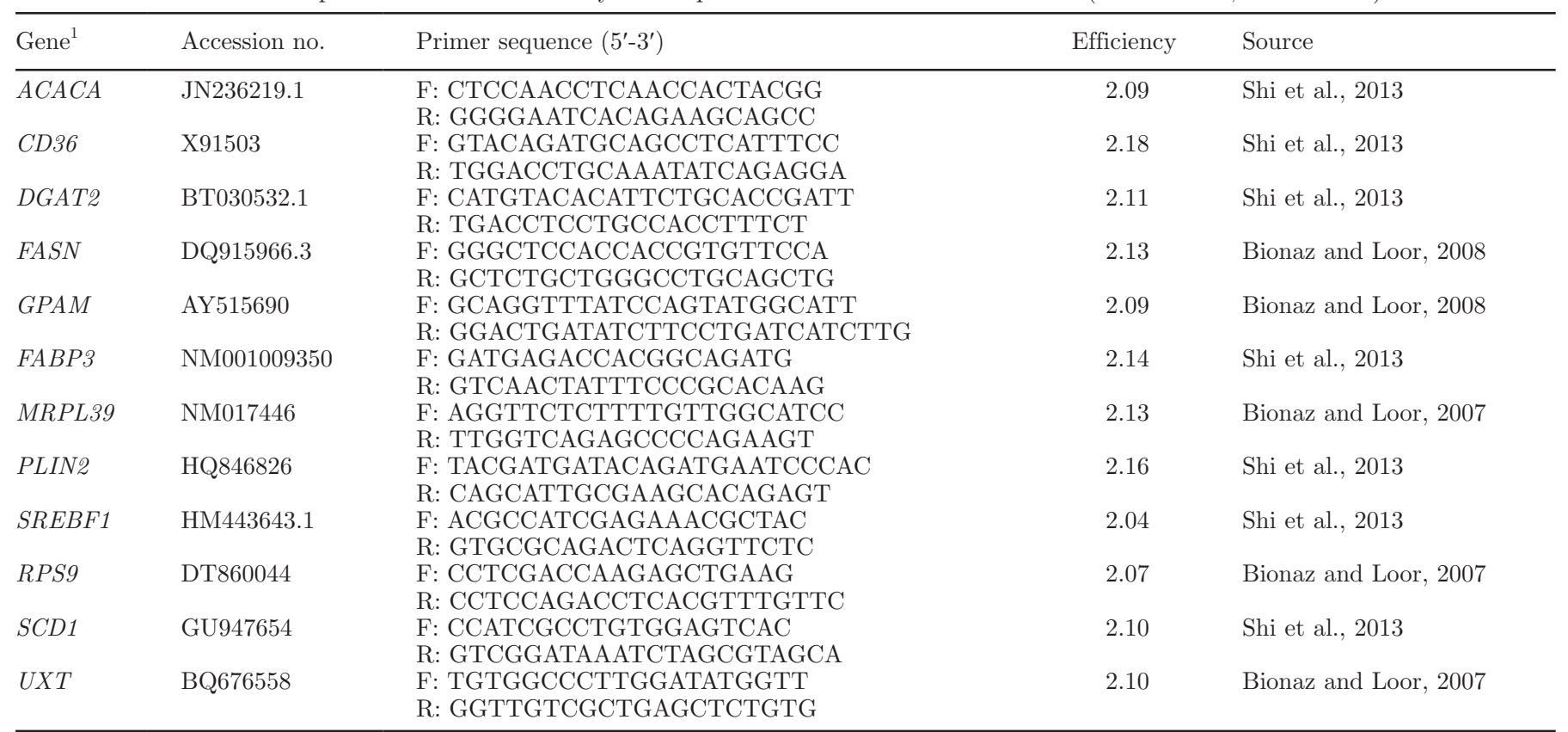

${ }^{1} A C A C A=$ acetyl-coenzyme A carboxylase $\alpha ; C D 36=$ thrombospondin receptor; $D G A T 2=$ diacylglycerol acyltransferase $2 ; F A S N=$ fatty acid synthase; GPAM = glycerol-3-phosphate acyltransferase; FABP3 = fatty acid binding protein 3; MRPL39= mitochondrial ribosomal protein L39; PLIN2 = perilipin 2; SREBF1 = sterol regulatory element binding transcription factor $1 ; R P S 9=$ ribosomal protein $\mathrm{S} 9 ; S C D 1=$ stearoylcoenzyme A desaturase 1; UXT = ubiquitously expressed transcript. 
A

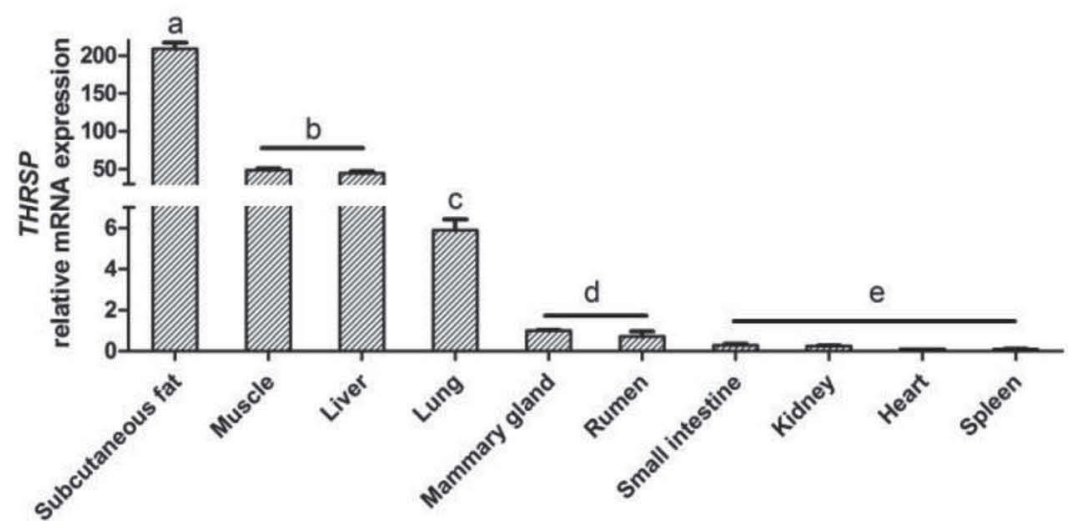

B

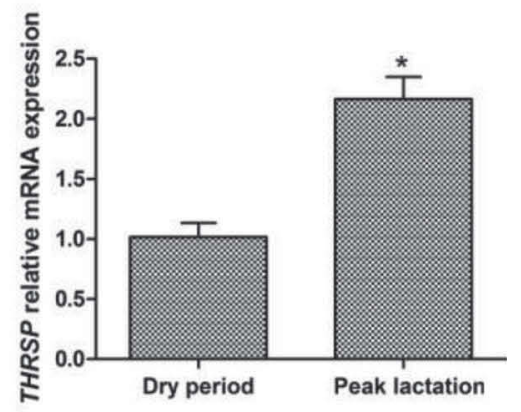

Figure 1. Expression pattern of thyroid hormone responsive (THRSP) in different goat tissues. (A) mRNA expression of THRSP in 10 different goat tissues. Dissimilar letters denote significant differences $(P<0.05)$ among tissues. $(\mathrm{B})$ mRNA expression of THRSP in mammary tissue from the dry period and peak lactation. *Significantly higher $(P<0.05)$ compared with the dry period. Values are means \pm SEM for 3 individuals.

ratio between the desaturation product and the sum of the product and the substrate (Loor and Herbein, 2003). Comparisons were performed by Student's $t$-test (unpaired and 2-tailed) for a one-way ANOVA. Significance was declared at $P<0.05$.

\section{RESULTS}

\section{Expression Patterns of THRSP in Various Tissues of Dairy Goat}

The THRSP gene was ubiquitously expressed in subcutaneous fat, muscle, liver, lung, mammary gland, rumen, small intestine, kidney, heart, and spleen. Among these tissues, subcutaneous fat had the highest expression of THRSP, followed by muscle, liver, and lung (Figure 1A). Expression was almost undetectable in small intestine, kidney, heart, and spleen. Although the relative expression of THRSP in mammary gland was extremely low, it increased significantly $(P<0.05)$ in peak lactation compared with the dry period (Figure 1B).

\section{Overexpression of THRSP Influences the Expression of Genes Related to FA Metabolism in GMEC}

Compared with that in negative control cells, the expression of THRSP increased markedly after the cells were infected with Ad-THRSP at $48 \mathrm{~h}(P<0.05$; Figure $2 \mathrm{~A})$. Overexpression of THRSP upregulated the expression of FASN $(P<0.05)$ and had no effect $(P>0.05)$ on $A C A C A$ (Figure 2B). We detected no significant differences $(P>0.05)$ in the expression of SREBF1 between the 2 groups. In contrast, the expression of $S C D 1$ was higher in the Ad-THRSP compared with the Ad-GFP group $(P<0.05$; Figure 2B). Overexpression of THRSP led to the increase in expression of DGAT2, GPAM, and PLIN2 $(P<0.05$; Figure $2 \mathrm{C})$. In addition, overexpression of THRSP downregulated CD36 $(P<$ 0.05 ), which is related to FA uptake, but had no effect on expression of $F A B P 3$, which is responsible for intracellular FA transport $(P>0.05$; Figure 2D).

\section{THRSP Overexpression Alters Content of the MCFA in GMEC}

To further investigate the effect of THRSP on FA synthesis in GMEC, the relative content of MCFA was measured. Although the total FA content was not influenced upon THRSP overexpression, the relative contents of $\mathrm{C} 12: 0(P<0.05$; Figure $3 \mathrm{~A})$ and $\mathrm{C} 14: 0(P$ $<0.05$; Figure 3B) were increased after GMEC were treated with Ad-THRSP for 48 h. However, no apparent changes were observed in $\mathrm{C} 16: 0(P>0.05$; Figure $3 \mathrm{C})$. There was a significant decrease in C18:0 FA upon THRSP overexpression $(P<0.05$; Figure $3 \mathrm{D})$.

\section{THRSP Overexpression Upregulates Concentration of C16:1 and C18:1 in GMEC}

The FA analysis revealed a marked increase in C16:1 and C18:1 concentrations $(P<0.05$; Figure 4A and 4B). Concomitant with changes in FA composition, the desaturation index of C18:1 was markedly increased $(P<0.05$; Figure 4D), whereas that of C16:1 did not change $(P>0.05$; Figure $4 \mathrm{C})$ upon THRSP overexpres- 
A

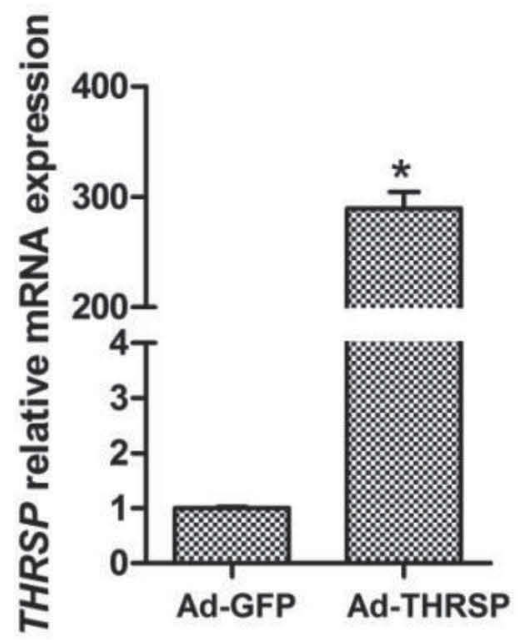

C TAG synthesis and lipid droplet formation

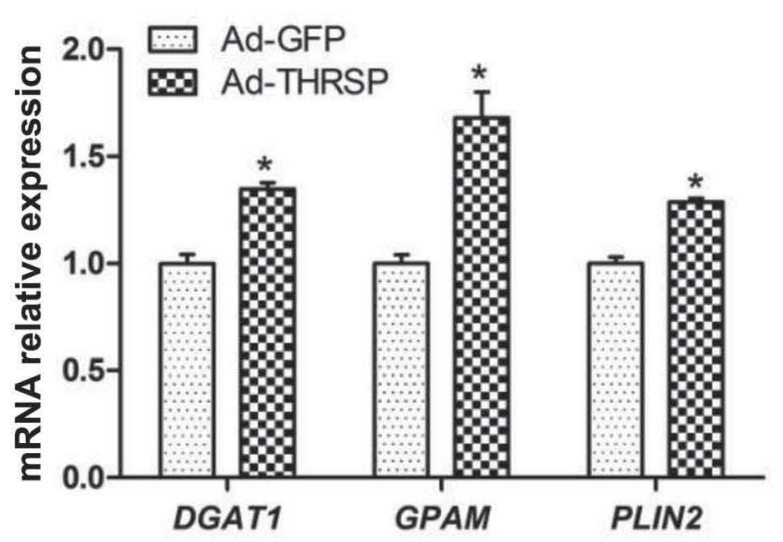

B de novo FA synthesis and desaturation

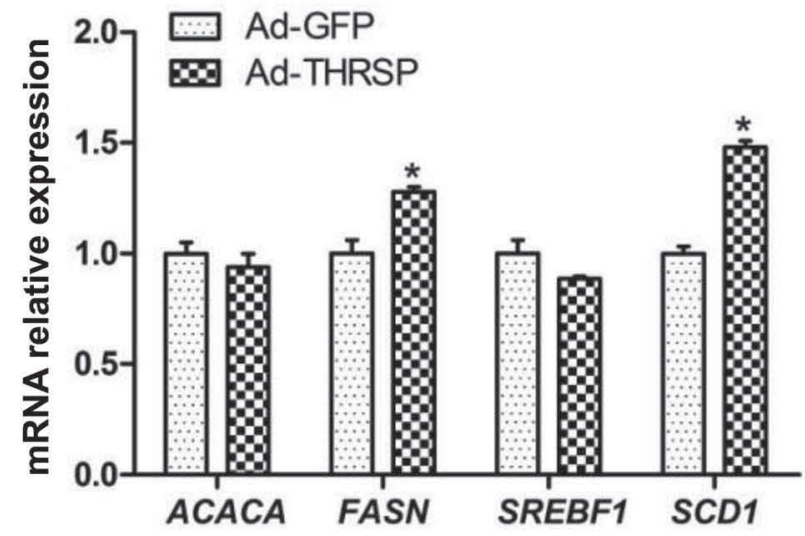

D $\quad$ FA uptake and transport

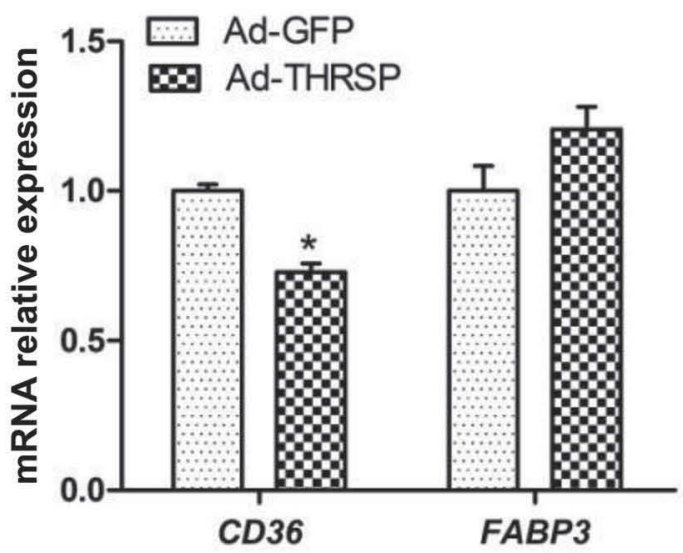

Figure 2. Overexpression of thyroid hormone responsive (THRSP) influences the expression of genes related to fatty acid (FA) metabolism in goat mammary epithelial cells (GMEC). (A) mRNA expression of THRSP in GMEC after a 48-h infection with Ad-GFP (control; adenovirus containing green fluorescent protein) or Ad-THRSP (adenovirus containing THRSP). (B) Effect of THRSP overexpression on expression of genes related to de novo fatty acid synthesis and desaturation (ACACA, FASN, SREBF1, SCD1). (C) Effect of THRSP overexpression on expression of genes related to FA uptake and transport (CD36 and FABP3). (D) Effect of THRSP overexpression on expression of genes related to triacylglycerol (TAG) synthesis and lipid droplet formation (DGAT2, GPAM, PLIN2). Data are means \pm SEM for 3 independent experiments; ${ }^{*} P<0.05$ vs. control (Ad-GFP). ACACA = acetyl-coenzyme A carboxylase $\alpha ; F A S N=$ fatty acid synthase; $S R E B F 1=$ sterol regulatory element binding transcription factor $1 ; S C D 1=$ stearoyl-coenzyme A desaturase $1 ; C D 36=$ thrombospondin receptor; FABP3= fatty acid binding protein 3; DGAT2 = diacylglycerol acyltransferase 2; GPAM = glycerol-3-phosphate acyltransferase; PLIN2 = perilipin 2.

sion. Furthermore, after overexpression of THRSP, intracellular TAG content increased markedly compared with the control group $(P<0.05$; Figure $4 \mathrm{~F})$.

\section{DISCUSSION}

The protein THRSP is located primarily in the nucleus, it is rapidly regulated by metabolic fuels and fuel-related hormones, and has been proven to be associated with FA synthesis in rodents (Cunningham et al., 1998). However, the role of THRSP in lactating mammary gland has not been fully resolved. The present study confirmed that THRSP is expressed predominantly in subcutaneous fat tissue and at much lower levels in liver and muscle. This pattern agrees with previous studies in mouse (Jump and Oppenheimer, 1985; Zhu et al., 2005) and cow (Harvatine and Bauman, 2006). The lower expression of THRSP in goat mammary gland, kidney, heart, and spleen is consistent with results in cow (Harvatine and Bauman, 
2006). The marked upregulation of THRSP at peak lactation compared with the dry period also agrees with the induction of THRSP mRNA in lactating rat mammary gland (Jump and Oppenheimer, 1985) and human mammary tissue (Moncur et al., 1998; Wells et al., 2006). Taken together, these findings raise the possibility that THRSP is an important lipogenic factor in lactating ruminant mammary gland.

The process of de novo lipogenesis in tissues such as mammary gland is closely correlated with multiple enzymes, including the rate-limiting enzyme ACACA and the terminal enzyme FASN (Clegg et al., 2001; Chajès et al., 2006). Accumulating evidence has suggested that THRSP may influence de novo FA synthesis through altering the activity or mRNA levels of these 2 enzymes. The first evidence comes from the reduction of FASN immunoreactivity upon Thrsp inhibition via antisense oligonucleotide in rat hepatocytes (Kinlaw et al., 1995). Hepatic overexpression of Thrsp in mouse revealed an upregulation of $F A S N$ expression along with a significant increase in the activity of FASN and ACACA (Wu et al., 2013). Those findings are further supported by the stimulation of in vitro activity of recombinant FASN by recombinant Thrsp, which suggests that Thrsp serves
A

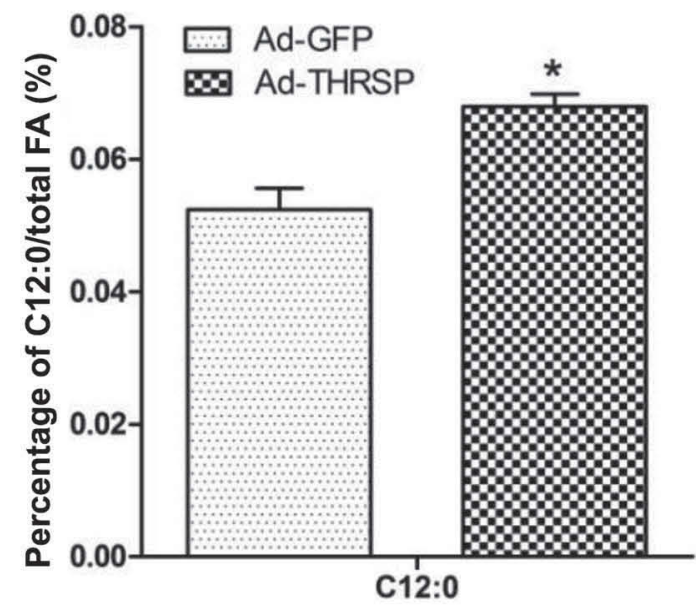

C

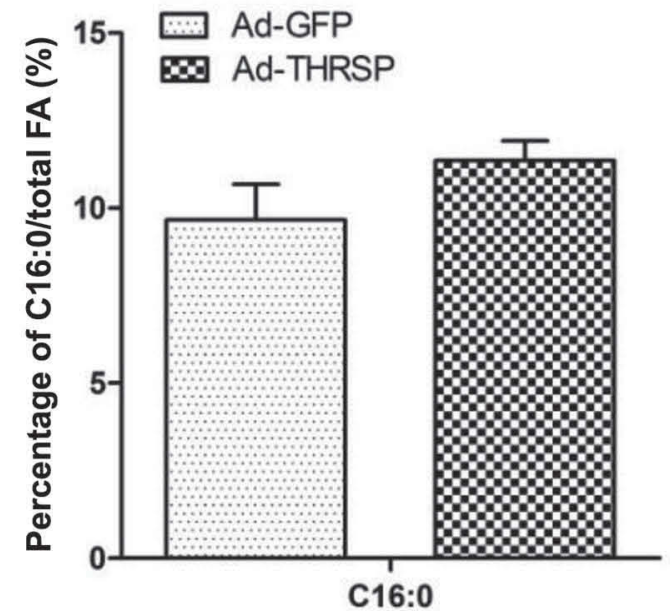

B

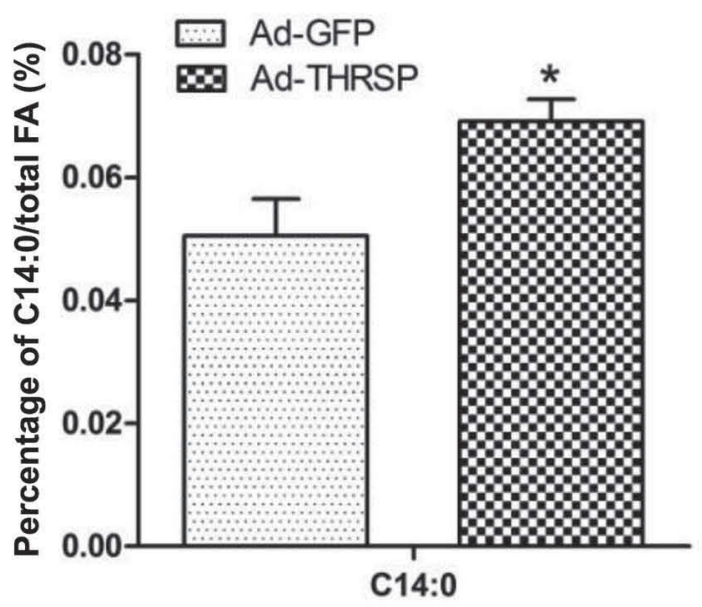

D

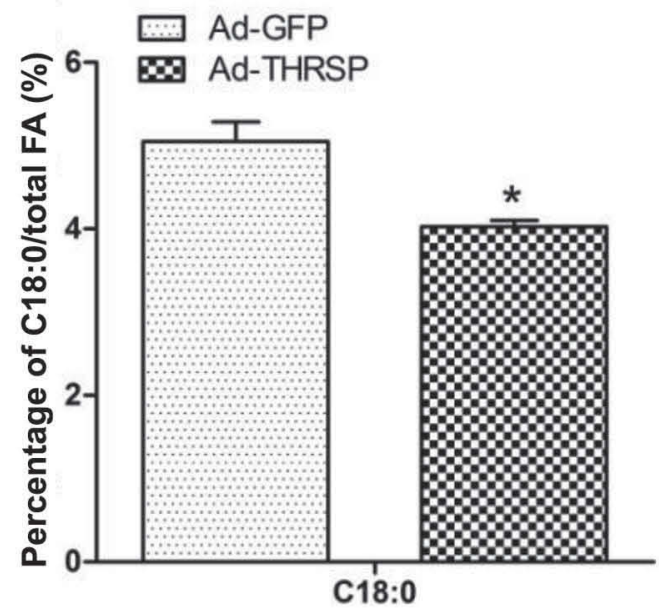

Figure 3. Overexpression of thyroid hormone responsive (THRSP) changes the fatty acid (FA) content in goat mammary epithelial cells (GMEC). Panels A, B, C, and D depict the difference in concentration of 12:0, 14:0, 16:0, and 18:0, respectively, in GMEC infected with AdTHRSP (adenovirus containing THRSP) compared with the control (Ad-GFP, adenovirus containing green fluorescent protein). Data are means \pm SEM for 3 independent experiments; ${ }^{*} P<0.05$ vs. control (Ad-GFP). 
A

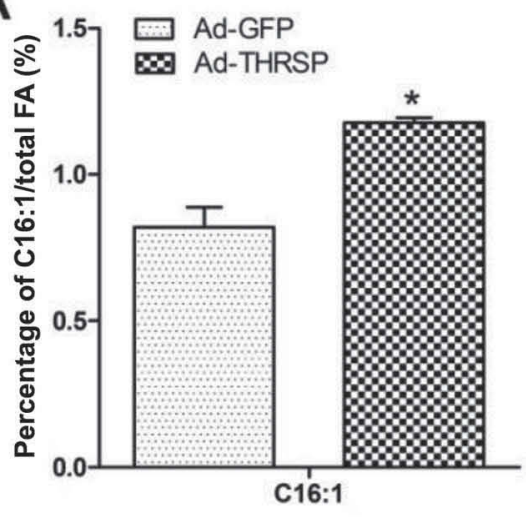

D

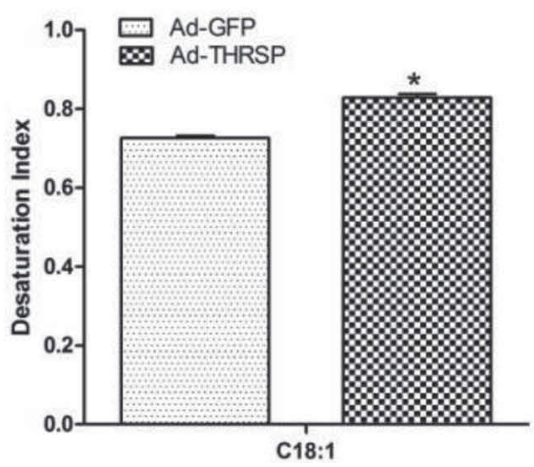

B

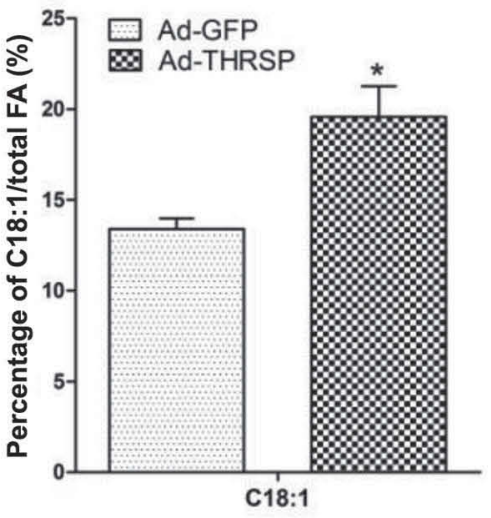

E

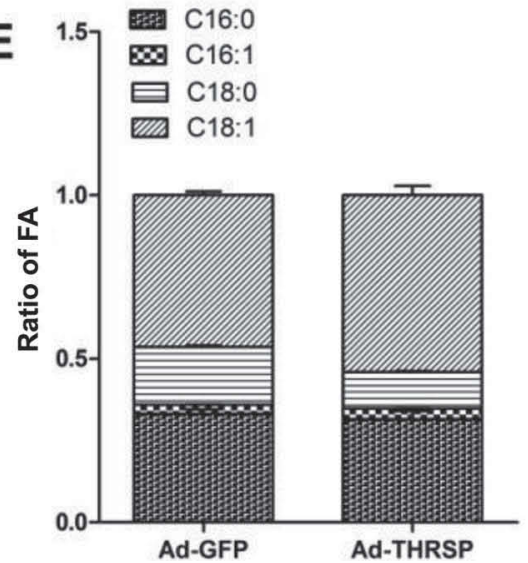

C

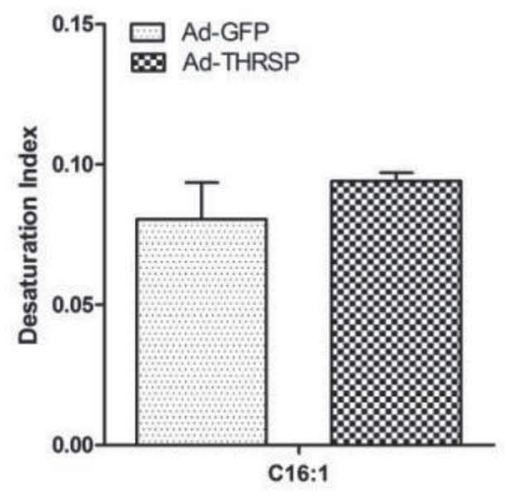

F

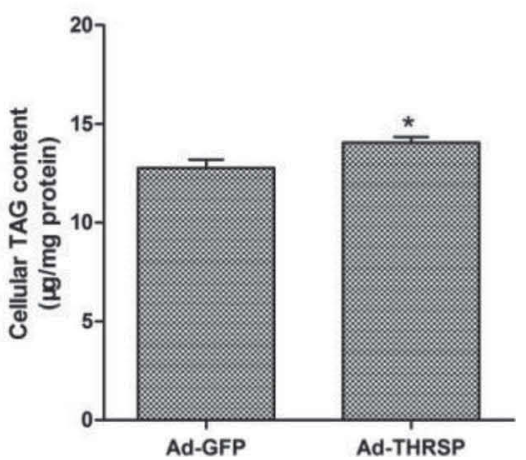

Figure 4. Overexpression of thyroid hormone responsive (THRSP) changes the intracellular fatty acid (FA) desaturation index and triacylglycerol (TAG) content in goat mammary epithelial cells (GMEC). Panel A, B, C, D, E, and F depict the difference in concentration of 16:0, 18:1, 16:1 desaturation index, 18:1 desaturation index, concentration of FA with 16- to 18-carbons, and total TAG, respectively, in GMEC infected with Ad-THRSP (adenovirus containing THRSP) compared with the control (Ad-GFP, adenovirus containing green fluorescent protein). Data are means \pm SEM for 3 individual experiments; ${ }^{*} P<0.05$ vs. control (Ad-GFP).

as an enhancer of FASN catalysis in mouse lactating mammary epithelium (Rudolph et al., 2014).

More recent work, however, did not detect differences in the gene or protein expression of ACACA and FASN resulting from Thrsp overexpression in mice (Wellberg et al., 2014). Furthermore, deletion of Thrsp did not reduce ACACA and FASN enzyme activities in mouse lactating mammary gland (Zhu et al., 2005). In the present study, we provide direct evidence that overexpression of THRSP significantly increased the mRNA level of FASN only, which is consistent with a previous study in cows (Cui et al., 2015). Apparent discrepancies in different tissues or cell types may be due to the function of THRSP on stabilization of FASN to enhance catalysis or to relieve allosteric inhibition instead of controlling mRNA expression or enzyme activity.

Our data also revealed that the expression of $S R E B F 1$ was not influenced by THRSP overexpression, which differs from the results in the cow (Cui et al., 2015). Al- though SREBF1 and THRSP have been demonstrated to be downregulated in diet-induced milk fat depression in dairy cows (Harvatine and Bauman, 2006), the existence of potential crosstalk between them is still unknown. Taken together, as in the cow and rodent, these results indicate that THRSP may play a role in lipogenesis in goat mammary gland.

A recent study confirmed that $F A S N$ is responsible for the regulation of cellular short and MCFA synthesis in GMEC (Zhu et al., 2014). Therefore, the increase in FASN expression upon THRSP overexpression may lead to an alteration of FA composition in GMEC. Such a response was confirmed by the increase in C12:0 and C14:0 upon THRSP overexpression, which agrees with results from murine mammary epithelial cells from Thrsp-transgenic mice (Rudolph et al., 2014). The decrease in de novo lipid synthesis in the lactating mammary gland and the lack of change in mRNA, protein, and hydrolase activity of thioesterase II (TEII) 
in the mammary epithelial cells from Thrsp-null mice also supports our findings (Zhu et al., 2005; Rudolph et al., 2014).

Because the function of the resident thioesterase domain from FASN depends entirely on its covalent linkage to FASN (Naggert et al., 1991), THRSP was hypothesized to stimulate the interaction of the resident thioesterase domain with the acyl carrier domain of FASN to form a complex promoting substrate channeling and release of FA products (Rudolph et al., 2014). The downregulation of $C D 36$, a major protein responsible for FA uptake across the plasma membrane (Steneberg et al., 2015), upon overexpression of THRSP was consistent with a recent study (Wu et al., 2013) and suggested that the decrease in FA uptake may be caused by an increase in FA synthesis. Similarly, the unchanged expression of FABP3 may be due to its function as intracellular transport of long-chain FA instead of MCFA (Cho et al., 2011). Collectively, these experiments suggest that THRSP may serve as an enhancer of FASN for the catalysis of MCFA synthesis in goat mammary gland.

The lack of change in C16:0 was in line with results in mice (Rudolph et al., 2014), but the marked decrease in C18:0 after THRSP overexpression was not. The enzyme encoded by $S C D 1$ plays a pivotal role in the synthesis of milk MUFA via catalyzing palmitic and stearic acid to palmitoleic and oleic acid by introducing a cis double bond between carbons 9 and 10 (Miyazaki and Ntambi, 2003). Thus, although upregulation of de novo FA synthesis may lead to an increase in concentration of C16:0, it also serves as substrate of $S C D 1$ for desaturation. The increase in concentration of C16:1 and C18:1 along with the desaturation index of C18:1 also substantiates the enhanced activity of SCD1 after THRSP overexpression. Taken together, these findings indicate that THRSP may stimulate FA desaturation via SCD1 to maintain an appropriate balance of intracellular FA in GMEC.

An increase in de novo FA synthesis and desaturation may lead to lipid accumulation in GMEC. As such, the increased concentration of $\mathrm{C} 16: 1$ and $\mathrm{C} 18: 1$, the products of $S C D 1$, which are indispensable components of TAG (Enoch et al., 1976), may contribute to lipid accumulation in GMEC. This idea is supported by the upregulation of GPAM and DGAT2, along with the greater concentration of TAG when THRSP was overexpressed. The former is responsible for the acylation of glycerol-3-phosphate to form lysophosphatidic acid, a rate-limiting step in the beginning of TAG synthesis (Gonzalez-Baró et al., 2007). The latter is an enzyme located close to SCD1 on the endoplasmic reticulum membrane (Man, 2006) and possesses greater affinity for MUFA synthesized by SCD1 (Meegalla et al., 2002). These results are consistent with data from mouse (Wu et al., 2013) and cow (Cui et al., 2015). Similarly, a strong accumulation of neutral lipid droplets was reported in MCF-7 upon THRSP overexpression (Sanchez-Rodriguez et al., 2005). In contrast, knocking down Thrsp in mouse mammary gland (Zhu et al., 2005; Rudolph et al., 2014) led to a decrease in TAG content. Overall, data demonstrate that THRSP is required for TAG accumulation in lactating mammary gland.

Although our study provided direct evidence that THRSP enhances de novo FA synthesis and lipid accumulation in goat mammary cells, some limitations need to be considered. First, the protein expression of the genes measured needs to be determined because the relative mRNA expression cannot fully reflect the variation in gene expression. Second, activities of the enzymes should be measured, potentially using tracers, to better evaluate and quantify rates of FA synthesis. Last, the mechanisms for how THRSP interacts with FASN or SCD1 to promote lipogenesis and desaturation warrant further studies.

\section{CONCLUSIONS}

The increase in medium-chain and unsaturated FA content demonstrated a direct role for THRSP in modulating de novo FA synthesis and desaturation in goat mammary cells. Thus, manipulating the in vivo expression of THRSP may serve as an important strategy to improve the quality of goat milk.

\section{ACKNOWLEDGMENTS}

This research was jointly supported by the Special Fund for Agro-scientific Research in the Public Interest (Beijing, China; 201103038) and the "Transgenic New Species Breeding Program of China" (Beijing, China; 2014ZX08009-051B).

\section{REFERENCES}

Alférez, M. J., M. Barrionuevo, I. Lopez Aliaga, M. Sanz-Sampelayo, F. Lisbona, J. Robles, and M. Campos. 2001. Digestive utilization of goat and cow milk fat in malabsorption syndrome. J. Dairy Res. 68:451-461.

Babayan, V. K. 1981. Medium chain length fatty acid esters and their medical and nutritional applications. J. Am. Oil Chem. Soc. $58: 49 \mathrm{~A}-51 \mathrm{~A}$

Bionaz, M., and J. J. Loor. 2007. Identification of reference genes for quantitative real-time PCR in the bovine mammary gland during the lactation cycle. Physiol. Genomics 29:312-319.

Bionaz, M., and J. J. Loor. 2008. Gene networks driving bovine milk fat synthesis during the lactation cycle. BMC Genomics 9:366.

Chajès, V., M. Cambot, K. Moreau, G. M. Lenoir, and V. Joulin. 2006. Acetyl-CoA carboxylase $\alpha$ is essential to breast cancer cell survival. Cancer Res. 66:5287-5294. 
Cho, K. H., M. Kim, G. Jeon, and H. Chung. 2011. Association of genetic variants for FABP3 gene with back fat thickness and intramuscular fat content in pig. Mol. Biol. Rep. 38:2161-2166.

Clegg, R., M. Barber, L. Pooley, I. Ernens, Y. Larondelle, and M. Travers. 2001. Milk fat synthesis and secretion: Molecular and cellular aspects. Livest. Prod. Sci. 70:3-14.

Colbert, C. L., C.-W. Kim, Y.-A. Moon, L. Henry, M. Palnitkar, W B. McKean, K. Fitzgerald, J. Deisenhofer, J. D. Horton, and H. J. Kwon. 2010. Crystal structure of Spot 14, a modulator of fatty acid synthesis. Proc. Natl. Acad. Sci. USA 107:18820-18825.

Cui, Y., Z. Liu, X. Sun, X. Hou, B. Qu, F. Zhao, X. Gao, Z. Sun, and Q. Li. 2015. Thyroid hormone responsive protein spot 14 enhances lipogenesis in bovine mammary epithelial cells. In Vitro Cell. Dev. Biol. Anim. 51:586-594.

Cunningham, B. A., J. T. Moncur, J. T. Huntington, and W. B. Kinlaw. 1998. "Spot 14" protein: A metabolic integrator in normal and neoplastic cells. Thyroid 8:815-825.

Enoch, H. G., A. Catala, and P. Strittmatter. 1976. Mechanism of rat liver microsomal stearoyl-CoA desaturase. Studies of the substrate specificity, enzyme-substrate interactions, and the function of lipid. J. Biol. Chem. 251:5095-5103.

Folch, J., M. Lees, and G. Sloane-Stanley. 1957. A simple method for the isolation and purification of total lipids from animal tissues. J. Biol. Chem. 226:497-509.

Gonzalez-Baró, M. R., T. M. Lewin, and R. A. Coleman. 2007. Regulation of triglyceride metabolism II. Function of mitochondrial GPAT1 in the regulation of triacylglycerol biosynthesis and insulin action. Am. J. Physiol. Gastrointest. Liver Physiol. 292:G1195G1199.

Haenlein, G. 2004. Goat milk in human nutrition. Small Rumin. Res. $51: 155-163$.

Haenlein, G., and R. Caccese. 1984. Goat milk versus cow milk. Page 1, E-1 in Goat Extension Handbook. G. F. W. Haenlein and D. L. Ace, ed. USDA Publ., Washington, DC.

Harvatine, K. J., and D. E. Bauman. 2006. SREBP1 and thyroid hormone responsive spot 14 (S14) are involved in the regulation of bovine mammary lipid synthesis during diet-induced milk fat depression and treatment with CLA. J. Nutr. 136:2468-2474.

Hinders, R. 1999. Special rations for specialty milks: High conjugated linoleic acid milk fat. Feedstuffs 71:18.

Jump, D. B., and J. H. Oppenheimer. 1985. High basal expression and 3,5,3'-triiodothyronine regulation of messenger ribonucleic acid S14 in lipogenic tissues. Endocrinology 117:2259-2266.

Kadegowda, A. K., M. Bionaz, L. Piperova, R. Erdman, and J. Loor. 2009. Peroxisome proliferator-activated receptor- $\gamma$ activation and long-chain fatty acids alter lipogenic gene networks in bovine mammary epithelial cells to various extents. J. Dairy Sci. 92:4276-4289.

Kang, Y., S. Hengbo, L. Jun, L. Jun, Z. Wangsheng, T. Huibin, and S. Huaiping. 2015. PPARG modulated lipid accumulation in dairy GMEC via regulation of ADRP gene. J. Cell. Biochem. 116:192201

Kim, C.-W., Y.-A. Moon, S. W. Park, D. Cheng, H. J. Kwon, and J. D. Horton. 2010. Induced polymerization of mammalian acetylCoA carboxylase by MIG12 provides a tertiary level of regulation of fatty acid synthesis. Proc. Natl. Acad. Sci. USA 107:9626-9631.

Kinlaw, W. B., J. L. Church, J. Harmon, and C. N. Mariash. 1995 Direct evidence for a role of the" spot 14" protein in the regulation of lipid synthesis. J. Biol. Chem. 270:16615-16618.

Kompan, D., and A. Komprej. 2012. The effect of fatty acids in goat milk on health. Pages 1-26 in Milk Production-An Up-To-Date Overview of Animal Nutrition, Management and Health. N. Chaiyabutr, ed. InTech, Croatia.

LaFave, L. T., L. B. Augustin, and C. N. Mariash. 2006. S14: Insights from knockout mice. Endocrinology 147:4044-4047.

Li, J., J. Luo, J. Zhu, Y. Sun, D. Yao, H. Shi, and W. Wang. 2015. Regulation of the fatty acid synthase promoter by liver X receptor $\alpha$ through direct and indirect mechanisms in goat mammary epithelial cells. Comp. Biochem. Physiol. B Biochem. Mol. Biol. 184:44-51
Loor, J. J., and J. Herbein. 2003. Reduced fatty acid synthesis and desaturation due to exogenous trans10, cis12-CLA in cows fed oleic or linoleic oil. J. Dairy Sci. 86:1354-1369.

Man, W. C. 2006. Colocalization of SCD1 and DGAT2: Implying preference for endogenous monounsaturated fatty acids in triglyceride synthesis. J. Lipid Res. 47:1928-1939.

Martel, P. M., C. M. Bingham, C. J. McGraw, C. L. Baker, P. M. Morganelli, M. L. Meng, J. M. Armstrong, J. T. Moncur, and W. B. Kinlaw. 2006. S14 protein in breast cancer cells: Direct evidence of regulation by SREBP-1c, superinduction with progestin, and effects on cell growth. Exp. Cell Res. 312:278-288.

Mather, I. H., and T. W. Keenan. 1998. Origin and secretion of milk lipids. J. Mammary Gland Biol. Neoplasia 3:259-273.

Meegalla, R. L., J. T. Billheimer, and D. Cheng. 2002. Concerted elevation of acyl-coenzyme A: Diacylglycerol acyltransferase (DGAT) activity through independent stimulation of mRNA expression of DGAT1 and DGAT2 by carbohydrate and insulin. Biochem. Biophys. Res. Commun. 298:317-323.

Miyazaki, M., and J. M. Ntambi. 2003. Role of stearoyl-coenzyme A desaturase in lipid metabolism. Prostaglandins Leukot. Essent. Fatty Acids 68:113-121.

Moncur, J. T., J. P. Park, V. A. Memoli, T. a. Mohandas, and W. B. Kinlaw. 1998. The "Spot 14" gene resides on the telomeric end of the 11q13 amplicon and is expressed in lipogenic breast cancers: Implications for control of tumor metabolism. Proc. Natl. Acad. Sci. USA 95:6989-6994.

Naggert, J., A. Witkowski, B. Wessa, and S. Smith. 1991. Expression in Escherichia coli, purification and characterization of two mammalian thioesterases involved in fatty acid synthesis. Biochem. J. 273:787-790.

Peterson, D. G., E. A. Matitashvili, and D. E. Bauman. 2004. The inhibitory effect of trans-10, cis-12 CLA on lipid synthesis in bovine mammary epithelial cells involves reduced proteolytic activation of the transcription factor SREBP-1. J. Nutr. 134:2523-2527.

Ramunno, L., G. Cosenza, A. Rando, A. Pauciullo, R. Illario, D. Gallo, D. Di Berardino, and P. Masina. 2005. Comparative analysis of gene sequence of goat CSN1S1 F and $\mathrm{N}$ alleles and characterization of CSN1S1 transcript variants in mammary gland. Gene 345:289-299.

Rudolph, M. C., J. L. McManaman, L. Hunter, T. Phang, and M. C. Neville. 2003. Functional development of the mammary gland: Use of expression profiling and trajectory clustering to reveal changes in gene expression during pregnancy, lactation, and involution. J. Mammary Gland Biol. Neoplasia 8:287-307.

Rudolph, M. C. E. A. Wellberg, A. S. Lewis, K. L. Terrell, A. L. Merz, N. K. Maluf, N. J. Serkova, and S. M. Anderson. 2014. Thyroid hormone responsive protein Spot14 enhances catalysis of fatty acid synthase in lactating mammary epithelium. J. Lipid Res. 55:1052-1065.

Sanchez-Rodriguez, J., J. p. Kaninda-Tshilumbu, A. Santos, and A. Perez-Castillo. 2005. The spot 14 protein inhibits growth and induces differentiation and cell death of human MCF-7 breast cancer cells. Biochem. J. 390:57-65.

Seelig, S., C. Liaw, H. C. Towle, and J. H. Oppenheimer. 1981. Thyroid hormone attenuates and augments hepatic gene expression at a pretranslational level. Proc. Natl. Acad. Sci. USA 78:4733-4737.

Shi, H., J. Luo, D. Yao, J. Zhu, H. Xu, H. Shi, and J. Loor. 2013. Peroxisome proliferator-activated receptor- $\gamma$ stimulates the synthesis of monounsaturated fatty acids in dairy goat mammary epithelial cells via the control of stearoyl-coenzyme A desaturase. J. Dairy Sci. 96:7844-7853.

Shi, H., H. Shi, J. Luo, W. Wang, A. B. Haile, H. Xu, and J. Li. 2014. Establishment and characterization of a dairy goat mammary epithelial cell line with human telomerase (hT-MECs). Anim. Sci. J. 85:735-743.

Steneberg, P., A. G. Sykaras, F. Backlund, J. Straseviciene, I. Soderstrom, and H. Edlund. 2015. Hyperinsulinemia enhances hepatic expression of the fatty acid transporter Cd36 and provokes hepatosteatosis and hepatic insulin resistance. J. Biol. Chem. 290:19034-19043. 
Wang, W., J. Luo, Y. Zhong, X.-Z. Lin, H.-B. Shi, J.-J. Zhu, J. Li, Y.-T. Sun, and W.-S. Zhao. 2012. Goat liver X receptor $\alpha$, molecular cloning, functional characterization and regulating fatty acid synthesis in epithelial cells of goat mammary glands. Gene 505:114-120

Wang, Z., J. Luo, W. Wang, W. Zhao, and X. Lin. 2010. Characterization and culture of isolated primary dairy goat mammary gland epithelial cells. Sheng Wu Gong Cheng Xue Bao 26:1123-1127.

Wellberg, E. A., M. C. Rudolph, A. S. Lewis, N. Padilla-Just, P. Jedlicka, and S. M. Anderson. 2014. Modulation of tumor fatty acids, through overexpression or loss of thyroid hormone responsive protein spot 14 is associated with altered growth and metastasis. Breast Cancer Res. 16:481.

Wells, W. A., G. N. Schwartz, P. M. Morganelli, B. F. Cole, J. J. Gibson, and W. B. Kinlaw. 2006. Expression of "Spot 14" (THRSP) predicts disease free survival in invasive breast cancer: Immunohistochemical analysis of a new molecular marker. Breast Cancer Res. Treat. 98:231-240.

Williams, C. M. 2000. Dietary fatty acids and human health. Ann. Zootech. 49:165-180.
Wu, J., C. Wang, S. Li, S. Li, W. Wang, J. Li, Y. Chi, H. Yang, X. Kong, and Y. Zhou. 2013. Thyroid hormone-responsive SPOT 14 homolog promotes hepatic lipogenesis, and its expression is regulated by Liver X receptor $\alpha$ through a sterol regulatory elementbinding protein 1c-dependent mechanism in mice. Hepatology $58: 617-628$.

Zhu, J. J., J. Luo, W. Wang, K. Yu, H. B. Wang, H. B. Shi, Y. T. Sun, X. Z. Lin, and J. Li. 2014. Inhibition of FASN reduces the synthesis of medium-chain fatty acids in goat mammary gland. Animal 8:1469-1478.

Zhu, Q., G. W. Anderson, G. T. Mucha, E. J. Parks, J. K. Metkowski, and C. N. Mariash. 2005. The Spot 14 protein is required for de novo lipid synthesis in the lactating mammary gland. Endocrinology 146:3343-3350.

Zhu, Q., A. Mariash, M. R. Margosian, S. Gopinath, M. T. Fareed, G. W. Anderson, and C. N. Mariash. 2001. Spot 14 gene deletion increases hepatic de novo lipogenesis. Endocrinology 142:4363-4370. 